\title{
Interval Type-2 Fuzzy Logic Controller to Control the Velocity and Angle of Inverted Pendulum
}

\author{
Anita Khosla ${ }^{1}$, Research Scholar \\ EEE Dept, Faculty of Engineering and Technology, ManavRachna International University, India \\ Email: anitakhosla.fet@mriu.edu.in \\ Leena G. ${ }^{2}$, Professor \\ EEE Dept, Faculty of Engineering and Technology, ManavRachna International University, India \\ Email: leenag.fet@mriu.edu.in \\ M. K. Soni ${ }^{3}$, Exe. Director and Dean, \\ Faculty of Engineering and Technology, ManavRachna International University, India \\ Email: ed.fet@mriu.edu.in
}

\begin{abstract}
Inverted Pendulum is a well established benchmark problem that produces many challenges to a control en gineer. It is a nonlinear, unstable, non minimumphase and under actuated system.Itrequires a controller which canadapt in different disturbance conditions and work appreciably well when compared to conventional controllers. In this paper Interval type-2 Fuzzy logic controllerfor inverted pendulum isdesigned.The objective of the proposed control technique is to develop the stability position of the pendulum. The optimal membership functions and the interference system are developed using IT2FLS. Using the IT2FLS, the position of the inverted pendulum is maintained towards the reference position. The proposed control techniqueis implemented in MATLAB/Simulink working platform and the control performances are evaluated. Then, the performance of proposed controller is evaluated and compared with the PI controller, Fuzzy controller andABC- FLC.
\end{abstract}

Index Terms- Inverted Pendulum, Angle, Velocity, ABC Algorithm and IT2FLS

\section{INTRODUCTION}

In the region of control systems [1], the inverted pendulum method is a normal problem. For assessing the justification of different kinds of control theories for mechanical systems, an inverted pendulum is familiar which is tough to be controlled by a valid time controller [2] [3]. An Inverted Pendulum is aneasy pendulum that is apprehended in an erect vertical position. It has a cart and a simple rod (with or without bob) [4]. Two equilibria, one of which is stable while the other is unstable [5] are found. Rotational single- arm pendulum, the double arm pendulum and the cart inverted pendulum, etc. [6] are the most well-known kinds of the inverted pendulum.

A multivariable, nonlinear, fast reaction, unsteady and high order system [7] [8] is the cart type inverted pendulum system. The general cart-double-pendulum system (CDPS) contains two-link manipulator mounted on a cart where only the cart is set in motion and the manipulator connects perform as a passive double pendulum [9]. In order toe xamine and validatedissimilar control methods for dynamic systems with higher-order nonlinearities [10], the DIP is appropriate. The normal frequency of double inverted pendulum depends on the measurement lengthwise of the pendulum [11]. Under put into action degree one, for a class of under activated systems, that is, the quantity of control contributions is one less than that of degrees of independence [12].

The control setback of inverted pendulum is an additionalcomplicated [13] [14]. Henceto control the inverted pendulum systems, several control methods has been applied such as feedback stabilization, energy based control, bang-bang control, sliding mode control, robust control, hybrid control, partial linearization, PD, PID, and MPC [15][16]. Moreover these control methods are used to control of rockets, robots, fast moving ground vehicles and anti-seismic controls for buildings [17]. In the inverted pendulum [18], the steadiness problem is more significant.

Stabilization control is unfeasible if the two pendulums contain the similar natural frequency [19] for a paralleltype double inverted pendulum system. For the double inverted pendulum system [20], there are two main problems in the design of stabilizing controllers. The initial is wavering the pendulum up from the hang up position to the upright vertical position. The next problem consists in stabilization of the inverted pendulum about its unsteady equilibrium point [21]. Single inverted pendulum plan a stabilizing controllerwhich is a distinctive problem in control system [22]. For planning and examining controllers for these systems [9], quite a few approaches have been successful. They are genetic algorithms, incomplete feedback linearization and integrator back stepping, energy based approach [23]. In order to control the angle and velocity variation of the inverted pendulum, an IT2FLC control technique is proposed in this paper. The proposed technique is detailed in following section. 
The paper is organized as follows. The pendulum model and clarification of the suggested control approach are explained in section II. IT2FLS is discussed in section III.In section IV results and discussions are given with performance analysis. The section $\mathrm{V}$ concludes the paper.

\section{MODELING OF INVERTED PENDULUM}

The inverted pendulum system is one of the examples which iscommonly used in control system application. The reputation of the drives in part from the fact that it is unstable without control. Because, the pendulum will simply fall over if the cart isn't moved to balance it. In addition, the dynamic states of the system are nonlinear. The intention of the control system based controller is to balance the inverted pendulum by applying a force to the cart that the pendulum is attached. The free body diagram is illustrated in Fig.2.

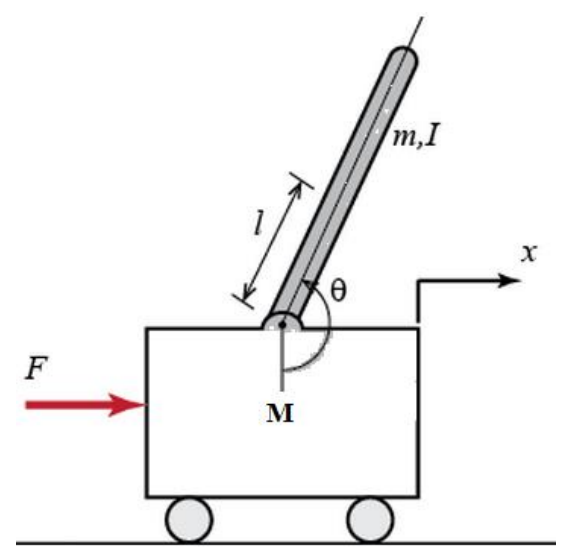

Fig. 1. Configuration of Inverted Pendulum

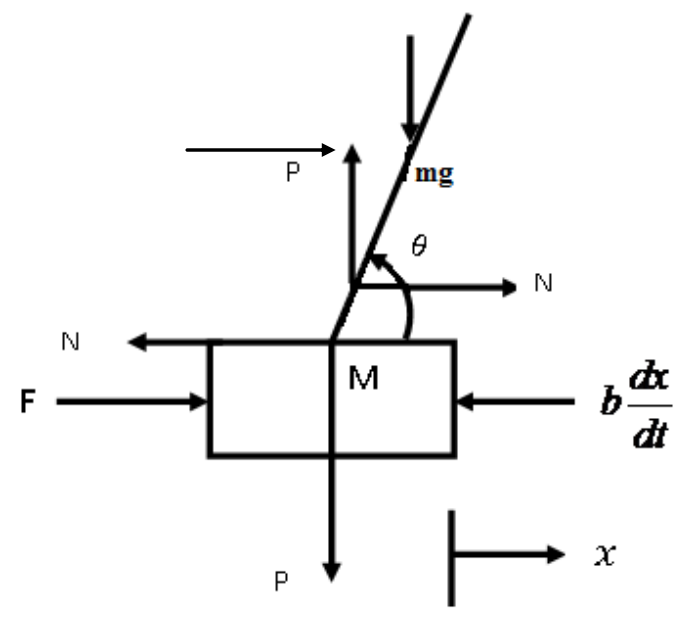

Fig. 2. Free body diagram

In the above diagram (Fig. 1 and Fig.2), $F$ is the force applied to the cart, $M$ is the mass of the cart, $m$ is the mass of the pendulum, $\theta$ is the angular position of the pendulum, $x$ is the horizontal position of the cart and $l$ is the length to pendulum center of mass.
From the free body diagram, the resultant force acting one the cart is obtained by summing the forces and the motion equation is described as follow:

$$
F=M \frac{d^{2} x}{d t^{2}}+b \frac{d x}{d t}+N
$$

Then, the governing force equation of the system is given below.

$$
\begin{gathered}
F-(M+m) \frac{d^{2} x}{d t^{2}}+b \frac{d x}{d t}+m l \frac{d^{2} \theta}{d t^{2}} \cos \theta- \\
l\left(\frac{d \theta}{d t}\right)^{2} \sin \theta
\end{gathered}
$$

To add the forces that acts perpendicular to the pendulum and getting the second motion equation of the system. Then, solving the system along with this axis greatly simplifies the mathematics and the present the equation as follow,

$$
\begin{gathered}
P \sin \theta+N \cos \theta-m g \sin \theta \\
=m \frac{d^{2} x}{d t^{2}} \cos \theta+m l \frac{d^{2} \theta}{d t^{2}}
\end{gathered}
$$

The centroid of the pendulum to get by adding the moments and the equation is described as below.

$$
-P l \sin \theta-N l \cos \theta=I \frac{d^{2} \theta}{d t^{2}}
$$

Then, combining the equation (3) and (4), get the second governing equation which is expressed as following them,

$$
\left(I+m l^{2}\right) \frac{d^{2} \theta}{d t^{2}}+m g l \sin \theta=-m l \frac{d^{2} x}{d t^{2}} \cos \theta
$$

As the analysis and the control design techniques of the linear systems, the set of equation needs to be linearized. The vertical upward equilibrium position, $\theta=\pi$ that are stays the system with the equilibrium position. This assumption should be reasonably valid while the pendulum not deviates from the desire position. Actually, the desired position of the pendulum not deviate more than $20^{\circ}$ from the vertical upward position. Let, $\phi$ indicate the pendulum deviation position from the equilibrium position i.e. $\theta=\pi+\phi$. After, applying the equilibrium condition, the equations are modified as follow,

$$
\left(\frac{d \theta}{d t}\right)^{2}=\left(\frac{d \phi}{d t}\right)^{2} \approx 0
$$

After substituting the approximations (equation (6), the governing equation of the systemis described as follow,

$$
m l \frac{d^{2} x}{d t^{2}}=\left(I+m l^{2}\right) \frac{d^{2} \phi}{d t^{2}}-m g l \phi
$$




$$
(M+m) \frac{d^{2} x}{d t^{2}}+b \frac{d x}{d t}-m l \frac{d^{2} \phi}{d t^{2}}=0
$$

The above mathematical derivations are used to model inverted pendulum. For controlling the angle and the velocity of the pendulum, the IT2FLS is used. While controlling the angle and velocity of the pendulum, optimized membership function is used as the input of the IT2FLS interference system.

Initially, the angle and velocity of the inverted pendulum are evaluated. After that, the change in the velocity and the change in angles are determined by taking the difference between the present and earlier error. By giving these calculated change of angle and change of velocity as the input to the IT2LS, the control output is calculated.

\section{CONTROLLINGTHEPENDULUM ANGLE AND VELOCITY USING IT2FLS}

Fuzzy logic controller is considered [29] to stabilize the inverted pendulum system. In this paper, IT2FLS is used for controlling the inverted pendulum angle and the velocity with minimu m control deviation. Interval Type 2 fuzzy logic system includes fuzzifier, rule base, inference engine, type reductions and defuzzifier [30]. In the IT2FLS, the rule base part enclosed with antecendents and consequents parts and each rule inference output is a type-2 fuzzy set. The membership function limits are chosen with the help of the optimal velocity and the optimal angle. It is used for reducing footprint of uncertainties (FOU). A uniform weighting is supposed to symbolize a FOU since the mean changeable between $\mathrm{m} 1$ and $\mathrm{m} 2$, the gaussian function is measured with a standard deviation. The structure of the propsoed IT2FLS is given in fig. 3

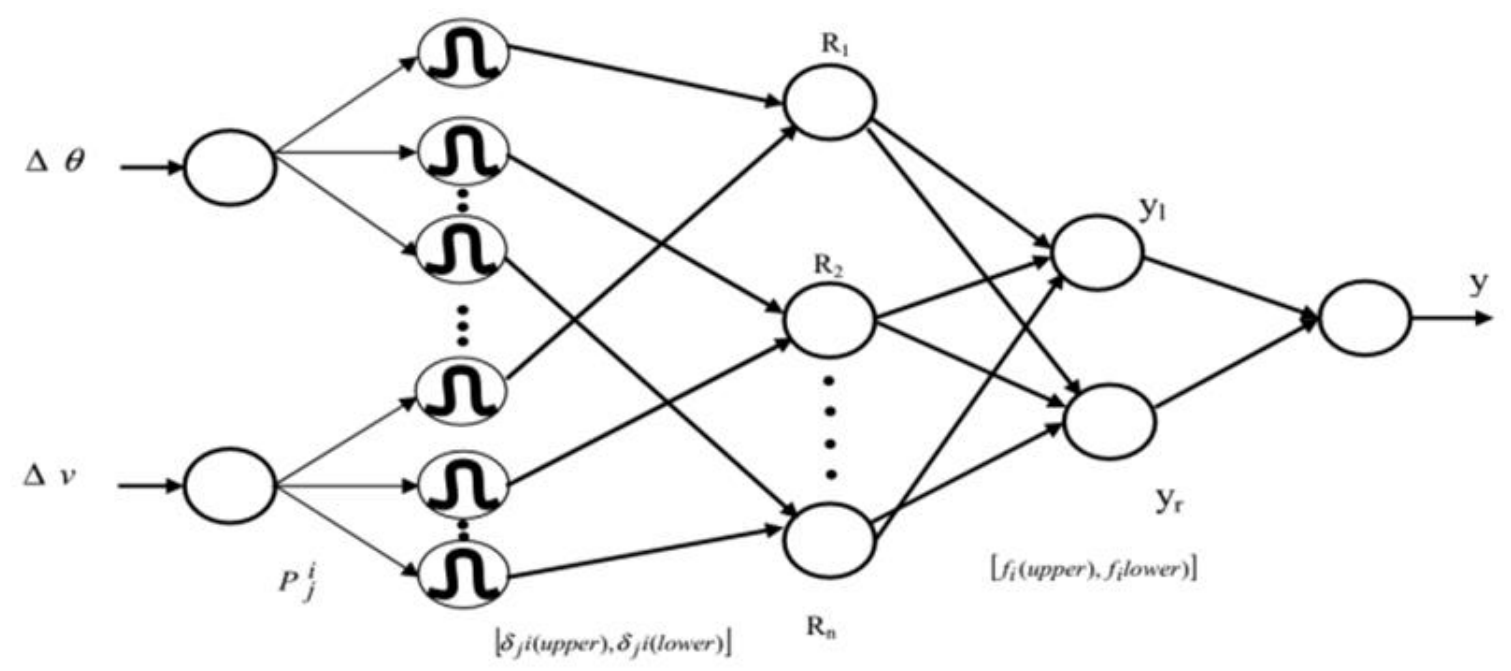

Fig. 3. Structure of proposed IT 2FLS

\section{Procedure of IT2FLS}

\section{Step 1: Fuzzification}

Fuzzification is the first step of the IT2FLS, in which the crisp parameters of input $\Delta \theta$ and $\Delta v$ are converted and fuzzified into input interval type-2 fuzzy sets. In fuzzification, the change of angle and change of velocity are mapped to the linguistic labels of fuzzy sets. The membership functions of these fuzzy sets contain two inputs and one output. Here, the input vector is $X_{i}=\left(x_{1}, x_{2}, \ldots \ldots x_{n}\right)$ and the if-then rules are specified in the following,

$$
\begin{aligned}
R l_{i}= & \text { If } x_{1} \text { is } P_{1}^{i} \text { AND } x_{2} \text { is } P_{2}^{i} \\
& \text {........... } \\
& \text { AND } x_{n} \text { is } P_{n}^{i} \text { THEN } Y^{i} \text { is } a_{i}
\end{aligned}
$$

Where, $P_{j}^{i}$ are the antecedents $j=1,2,3 \ldots . n$ and $Y_{i}$ is the consequent of the $i^{\text {th }}$ rule. Then the Gaussian membership function (MF) is used which has a fixed standard deviation (SD) and uncertain mean that takes in the values in $\left\lfloor m_{j 1}, m_{j 2}\right\rfloor$.The uncertainty of this membership functions can be represented in the bounded interval in terms of upper MF and lower MF. The Gaussian membership functions with uncertain mean described as,

$$
\delta_{j}^{i}\left(x_{j}\right)=\exp \left(\frac{-\left(x_{j}-m_{j}^{i}\right)^{2}}{2\left(D_{j}^{i}\right)^{2}}\right)
$$

Where, $m_{j}^{i} \in\left[m_{j 1}, m_{j 2}\right]$ and $j=1,2,3 \ldots . . n, n$ is the number of antecedents. Then the $i=1,2,3 \ldots . r, r$ is the number of rules. Then the upper and lower MF's are specified as $\delta_{j} i$ (upper) and $\delta_{j} i$ (lower) respectively and described in the equation (14) and (15).

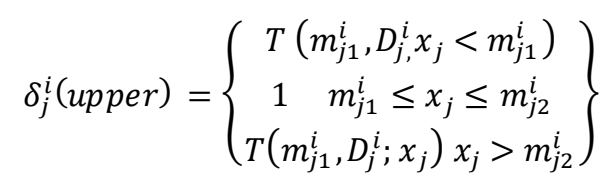




$$
=\left\{\begin{array}{l}
\delta_{j}^{i}\left(m_{j 2}^{i}, D_{j}^{i} ; x_{j}\right) x_{j} \leq \frac{m_{j 1}^{i}+m_{j 2}^{i}}{2} \\
T\left(m_{j 1}^{i}, D_{j}^{i} ; x_{j}\right) x_{j}>\frac{m_{j 1}^{i}+m_{j 2}^{i}}{2}
\end{array}\right\}
$$

Where, the output of the each node is represented as in the lower and upper interval.

\section{Step 2: Rule base system}

Here, by generating the rulethe fuzzy meet operation is done. For each node, two rules are generated by adding the upper membership functions seperately and lower membership function seperately. The output of a rule node is a firing strength $F S^{i}$, which is an IT2 fuzzy set. The firing strength is computed as follows,

$$
F^{i}=\left(f_{i}(\text { lower }), f_{i}(\text { upper })\right)
$$

Where,

$$
\begin{aligned}
& f_{i}(\text { upper })=\prod_{j=1}^{n} \delta_{j}^{i}(\text { upper }) \\
& f_{i}(\text { lower })=\prod_{j=1}^{n} \delta_{j}^{i}(\text { lower })
\end{aligned}
$$

\section{Step 3: Inference Engine}

Once the firing strength is obtained, the inference engine and the rule base are activated by the interval type-2 fuzzy sets to produce output. Then the input interval type- 2 fuzzy sets and output interval type- 2 are mapped by combining the fired rules using inference engine. Then outcome of the inference engine are processed by the type-reducer. The type-reducer combines the output sets and performs a centroid calculation which leads to type-1 fuzzy sets called the type-reduced sets.

\section{Step 4: Type Reductions}

The type reduced set of the interval type-2 FS is an interval type 1 set which contains the left limit yl and right limit yr. The interval output of this layer is computed by each node present in this layer. The consequent $a_{i}$ is a crisp value. Here we used a simplified type-2 reduction operation instead of the center-of-sets type reduction method. It considers only two embedded type-1 fuzzy sets with membership values, that is the outputs $y^{q}$ and $y^{s}$ are computed as follows,

$$
y^{q}=\frac{\sum_{i=1}^{M} f_{i}(\text { lower }) a_{i}}{\sum_{i=1}^{M} f_{i}(\text { lower })}
$$

$$
y^{S}=\frac{\sum_{i=1}^{M} f_{i}(\text { upper }) a_{i}}{\sum_{i=1}^{M} f_{i}(\text { upper })}
$$

After the type-reduction process, the type-reduced sets are then defuzzified.

\section{Step 4: Defuzzification}

It is the last process of the IT2FLS where each output node corresponds to one output variable. Due to the output layer of an interval set, each and every node performs defuzzification process. The defuzzified output is denoted by $\mathrm{y}$ which is determined by taking the average of the nodes $y^{q}$ and $y^{s}$.

$$
y=\frac{y^{q}+y^{s}}{2}
$$

Subsequently the obtained defuzzified control output of fuzzy system is given to inverted pendulum to reduce the deviations of the angle and the velocity of the pendulum and also the position of the pendulum are maintained that was close to the reference position.

\section{RESULTS AND DISCUSSION}

The proposed IT2FLS was implemented in MATLAB/simulink working platform. The performance of the proposed IT2FLS was tested with inverted pendulum cart system. Here, the change of velocity and angles are calculated from their difference between the present state error and the previous error which are given to the IT2FLS. In the IT2FLS, the uncertainties are reduced and the optimal membership functions are determined. By using IT2FLS, the control output is generated for the inverted pendulum, which is controlling the angle and the velocity also, the stability position of the pendulum are determined. The performances of the proposed technique are evaluated and compared with PI controller, Fuzzy controller and ABC-Fuzzy controller[24].

\subsection{Performance analysis}

Initially, the angles and velocity of the inverted pendulum performances are evaluated in normal position and illustrated in fig. 4(a) and 4(b) respectively. The output of IT2FLS is applied to the pendulum for controlling the angle and velocity. After that, evaluated the performances of the proposed method and illustrated in the figure 6. The performance of IT2FLS is illustrated in the fig. 6(a) and 6(b) respectively. Similarly, all the other methods were applied and illustrated the performance measures. 


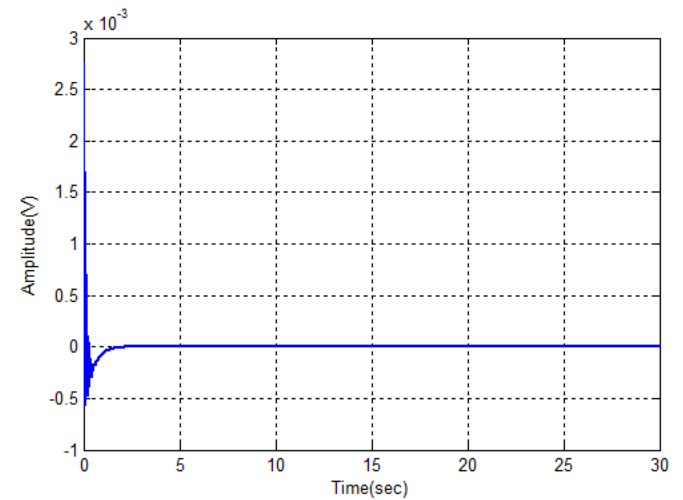

Fig 4(a). Performance of change of angle of inverted pendulum

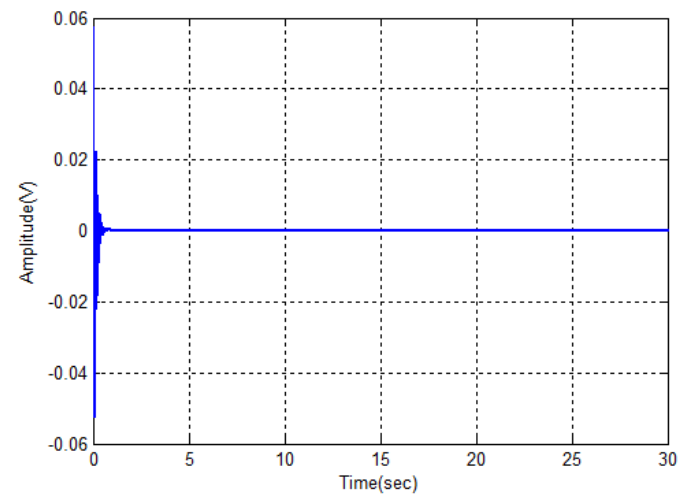

Fig.4(b). Performance of change of velocity of inverted pendulum

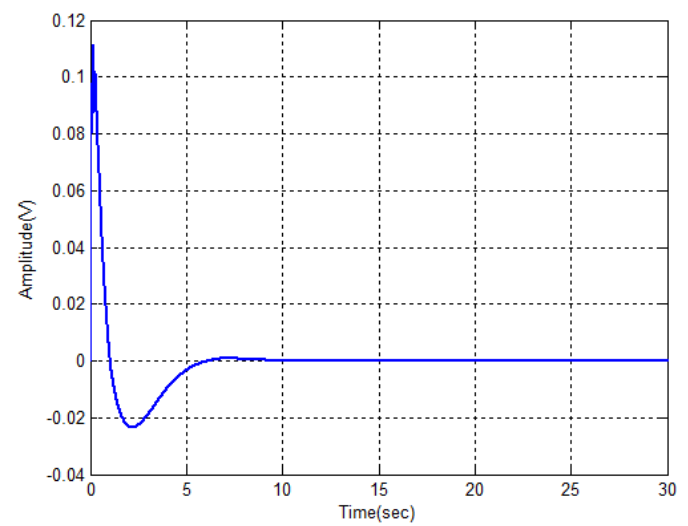

Fig. 5(a). Performance of pendulum angle using ABC-Fuzzy Controller

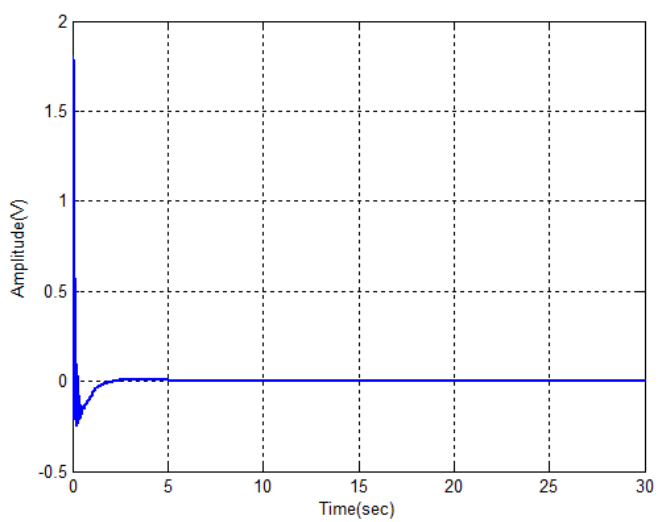

Fig.5(b). Performance of pendulum velocity using ABC-Fuzzy Controller.

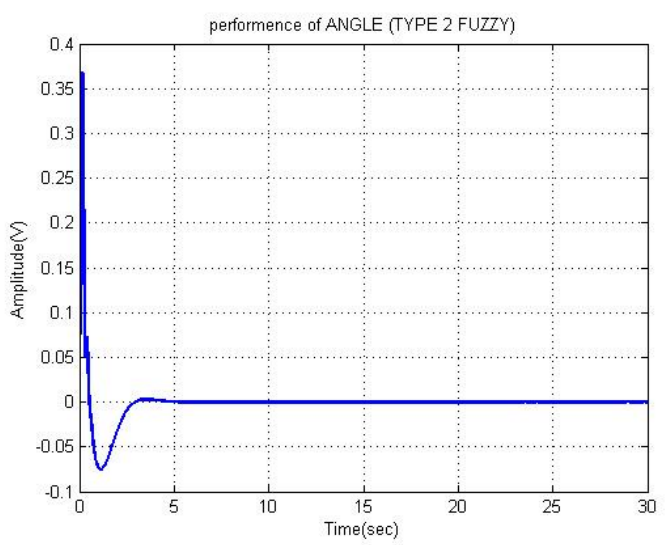

Fig. 6(a). Performance of pendulum angle using IT2FLS

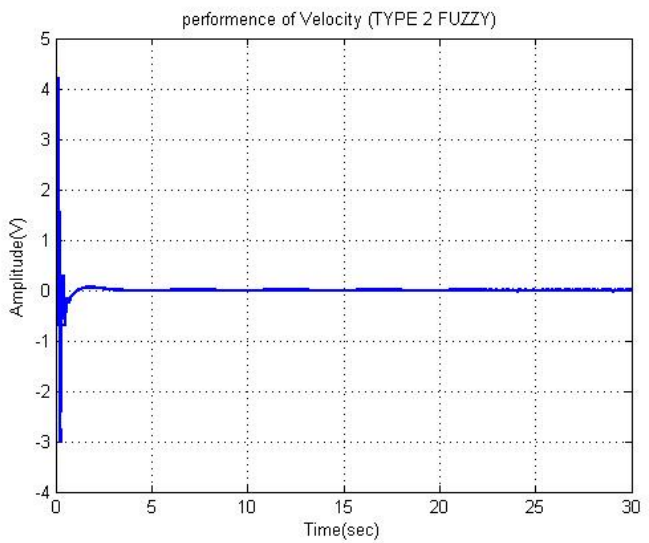

Fig. 6(b). Performance of pendulum velocity usingIT2FLS

From the above illustrations, the optimal value of pendulum angle and velocity are measured with the different time instants. Here, the various methods are used to calculate the optimal value of angle and velocity. The change of pendulum angle and the velocity is optimized with the time instant $\mathrm{T}=7 \mathrm{sec}$ and $\mathrm{T}=3 \mathrm{sec}$ respectively. Similarly, the optimized angle and velocity values are calculated in the fuzzy controller and IT2FLS. Therefore, the proposed controller achieves less time for optimizing the pendulum angle and velocity with compared to the other methods.

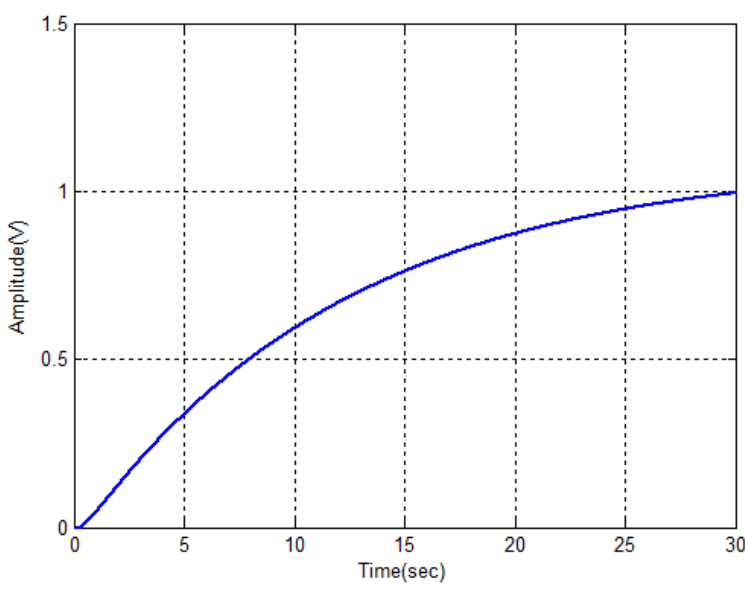

Fig.7. Performance of normal cartposition

I.J. Intelligent Systems and Applications, 2014, 07, 44-51 


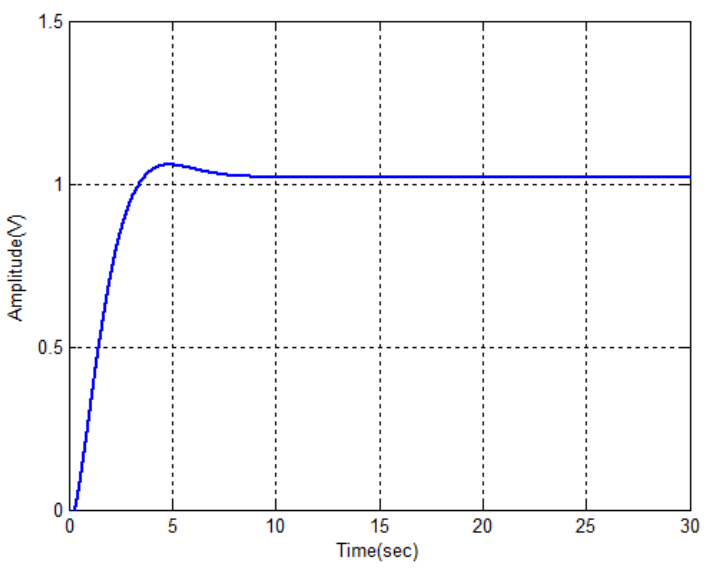

Fig. 8. Performance of Fuzzy cart position

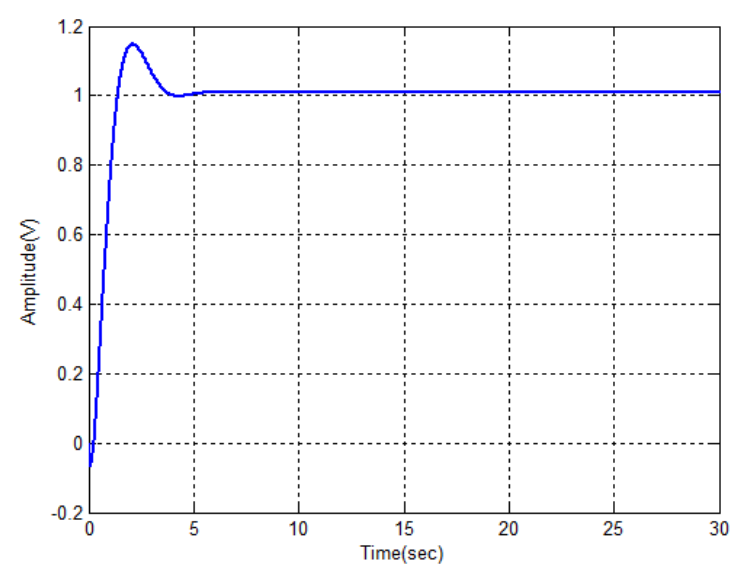

Fig.9. Performance of ABC-Fuzzy cart position

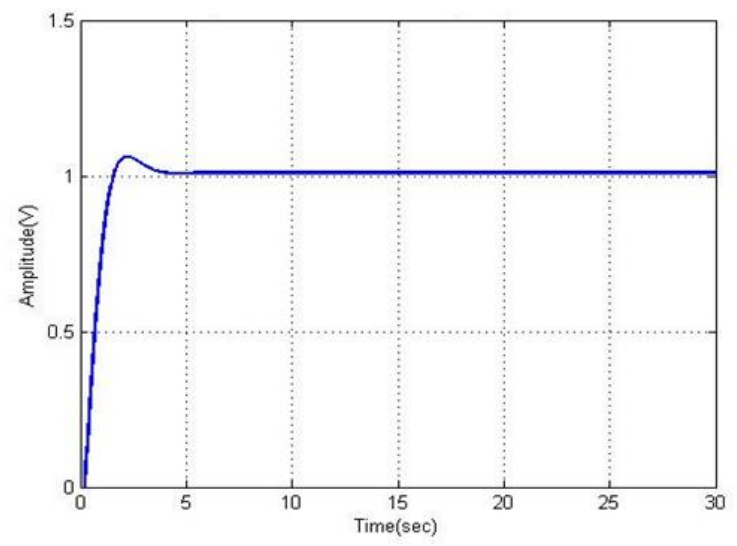

Fig. 10. Performance of IT 2FLS cart position

The performance of the normal cart position is illustrated in the fig. 7. After applying various methods, the performances of the cart positions are varied with different amplitude. Here, the Fuzzy controller, ABCFuzzy controller and IT2FLS methods are applied for analyzing performances of the cart positions, which are illustrated in the fig. 8,9 and 10 respectively. Similarly, the performances of the cart positions are measured by using PI controller. The performance comparison results of existing methods with the proposed method shows that the proposed method outperforms the existing methods by analyzing the cart positions within a short-span of time and small amplitude. Moreover, the rising time, settling time and peak time of the pendulum are evaluated for the proposed as well as existing methods.

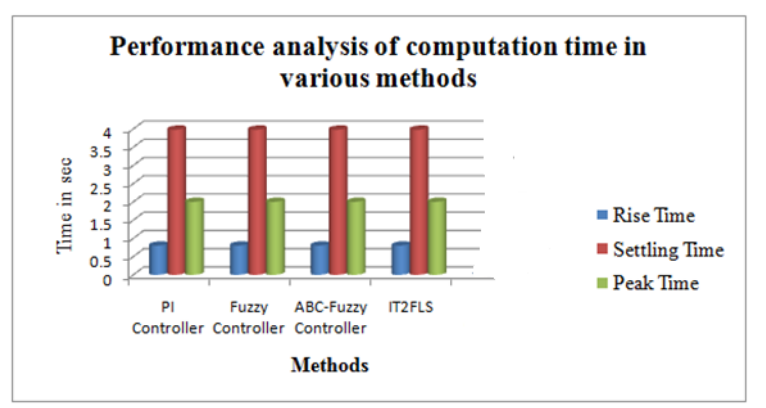

Fig. 11. Performance analysis of computation time in various methods

From the above illustration (fig. 11) describes the rising time, settling time and peak time of the PI controller, fuzzy controller, ABC-Fuzzy controller and IT2FLS respectively. Here, the peak value and peak time is not varied for most of these methods. The settling time of the proposed controller is $2.9800 \mathrm{sec}$. The settling time of the PI controller, Fuzzy controller and IT2FLS is denoted as $3.9781 \mathrm{sec}, 3.9782 \mathrm{sec}$, and $3.9781 \mathrm{sec}$ respectively. So, the proposed controller reaches the settling time to take very less time when compared to the other controller. The proposed method reaches the actual system state very quickly. But, the PI controller takes more time to reach the settling time and the fuzzy controller deviated from the stability control position. Also, the proposed control technique maintains the system stability while changing the system mass. Then the rising time of the PI controller, Fuzzy controller, ABC-Fuzzy controller and IT2FLS are measured with their performances. Here, the rising time of the proposed controller is less than the other methods. Therefore, the proposed technique achieves the better performances when compared to other techniques. Similarly, the peak time is also measured in every method.

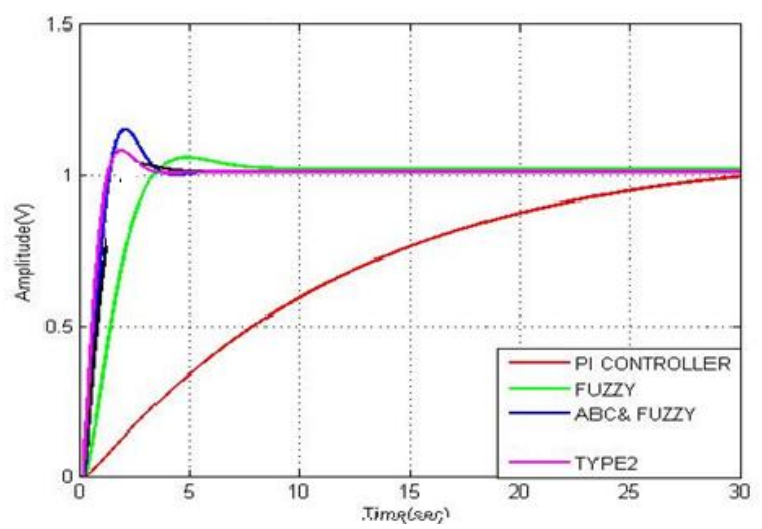

Fig. 12. Comparison performance of cart position of PI, Fuzzy, ABCFuzzy controller and proposed hybrid method

By using the proposed method, the stability cart position of the pendulum is analyzed. The performance of cart position after applying the proposed IT2FLC is described in Fig.10. Then, applying PI controller, fuzzy 
controller and ABC-Fuzzy controller, the stability performance of cart position is analyzed. The performance of proposed IT2FLC control approach is compared with PI controller, fuzzy controller andABCFuzzy controller. The comparis on performance of cart position is illustrated in Fig.12. From the above illustrations, the proposed controller (IT2FLS) is the effective method for controlling the angle and the velocity deviation of the pendulum compared with the other methods. Therefore, the stability of the cart position is steadily preserved in the proposed method with compared to the PI controller, fuzzy controller andABCFuzzy controller.

\section{CONCLUSION}

In the paper, a IT2FLC was proposed for controlling the angle and velocity of the inverted pendulum. The proposed technique was implemented in the MATLAB/Simulink platform. Then, the performance of the proposed controller was compared with PI controller, fuzzy controller, and ABC-Fuzzy controller. The settling time, rising time and peak time of the proposed controller and other methods were determined. The comparative analysis shows the proposed controller effectively control the change of angle and velocity of pendulum. So, the steady state stability of the cart position of the pendulum is maintained better than PI controller, fuzzy controller and ABC-Fuzzy controller

\section{REFERENCES}

[1] Arslan ZIA, Fahim G. Awan and Hasan. A. Nasir, "Implementation and Real-time Tuning of PID Control for Inverted Pendulum System with Time Delay - A Practical Approach", In proceedings of 9th international conference on computing, communications and control technologies, Orlando, Florida, USA, 2011.

[2] ShubhobrataRudra and Ranjit Kumar Barai, "Robust Adaptive Backstepping Control of Inverted Pendulum on Cart System", International Journal of Control and Automation, Vol. 5, No. 1, March 2012.

[3] A.Tahir and J.Yasin,'Implementation Of Inverted Pendulum Control, Plunks On Miscellaneous Tactics", International Journal of Electrical \& Computer Sciences, Vol.12, No.4, 2012.

[4] I. Hassanzadeh, A. Nejadfardand M . Z adi, "A Multi variable Adaptive Control Approach for Stabilization of a Cart-Type Double Inverted Pendulum", Hindawi publishing Corporation Mathematical Problems in Engineering, 2011.

[5] WaelBenrejeb and Olfaboubaker, "FPGA modeling and real-time embedded control design via LAVIEW software: application for swinging-up a pendulum", International Journal on smart sensing and intelligent systems, Vol. 5, No. 3, September 2012.

[6] Mohan Akole and BarjeevTyagi, "Design Of Fuzzy Logic Controller For Nonlinear Model Of Inverted PendulumCart System", In Proceedings Of 32nd National Systems Conference, NSC, December 2008.

[7] Yuan Shaoqiang, Liu Zhong and Li Xingshan, "Modeling and Simulation of Robot Based on Matlab/SimMechanics",
In Proceedings of the $27^{\text {th }}$ Chinese Control Conference, July 2008.

[8] Tung-Kuan Liu, Chiu-Hung Chen, Zu-Shu Li and JyhHorng Chou, "Method of Inequalities-based Multi objective Genetic Algorithm for Optimizing a Cart-doublependulum System", International Journal of Automation and Computing, Vol. 6(1), pp. 29-37, February 2009.

[9] Wei Zhong and Helmut Rock, "Energy and Passivity Based Control of the Double Inverted Pendulum on a Cart", In Proceedings of IEEE International Conference on Control Applications, Mexico city, sep , 2001.

[10] JianqiangYia, NaoyoshiYubazakib, and Kaoru Hirotac, "Anew fuzzy controller for stabilization of parallel-type double inverted pendulum system", International Journal on Fuzzy Sets and Systems,Vol.126, Issue 1, pp.105-119, February 2002.

[11] K.J. AsstroKm and K. Furuta, "Swinging up a pendulum by energy control", International Journal of Automatica, Vol. 36, pp.287-295, 2000.

[12] I. Hassanzadeh, A. Nejadfardand M . Z adi, "A Multi variable Adaptive Control Approach for Stabilization of a Cart-Type Double Inverted Pendulum”, Hindawi publishing Corporation Mathematical Problems in Engineering, 2011.

[13] Thamer Ali Albahkali ," An Impulse-Momentum Approach to Swing-Up Control of Double Inverted Pendulum on a Cart", World Academy of Science, Engineering and Technology, vol. 54, 2011.

[14] Mingcong Deng, Akira Inoue and Masaaki Kosugi," Swing-Up Control Of A Cart-Type Single Inverted Pendulum With Parasitic Dynamics", International Journal of Innovative Computing, Information and Control, Vol.3, No.6, pp. 1501-1510, December 2007.

[15] RagnarEide, Per Magne Egelid, Alexander Stams $\varnothing$ and Hamid Reza Karimi, "LQG Control Design for Balancing an Inverted Pendulum Mobile Robot", Journal of Intelligent Control and Automation, Vol.2, No.2, pp.160166, 2011.

[16] F. Chetouane , Member, Iaeng, S. Darenfed, and P. K. Singh, "Fuzzy Control of a Gyroscopic Inverted Pendulum", Journal of International Association of Engineers, 2010.

[17] A.Tahir and J.Yasin,'Implementation Of Inverted Pendulum Control, Plunks On Miscellaneous Tactics", International Journal of Electrical \& Computer Sciences, Vol.12, No.4, 2012.

[18] HenrikNiemann and JesperKildegaardPoulsen, "Analy sis and Design of Controllers for a Double Inverted Pendulum", In Proceedings of the American Control Conference Denver, Colorado, June 2003.

[19] D. Guida, F. Nilvetti and C.M. Pappalardo, "Dry friction of bearings on dynamics and control of an inverted pendulum", Journal of Achievements in Materials and Manufacturing Engineering, Vol. 38, Issue 1, January 2010.

[20] Sandeep Kumar Yadav, Sachin Sharma and Narinder Singh, "Optimal Control of Double Inverted Pendulum Using LQR Controller”, International Journal of Advanced Research in Computer Science and Software Engineering, Vol.2, Issue 2, pp. 189-192, February 2012.

[21] Mustafa Demirci, "Design Of Feedback Controllers For Linear System With Applications To Control Of A Double-Inverted Pendulum", International Journal of Computational Cognition, Volume 2, Number 4, Pages 65-84, December 2004.

[22] V. Casanova, J. Salt, R. Piza and A. Cuenca, "Controlling the Double Rotary Inverted Pendulum with Multiple 
Feedback Delays", International Journal of Computers, Communications \& Control, Vol.7, No.1, pp. 20-38, March 2012.

[23] Amanda Young, Chengyu Cao, Naira Hovakimy an and Eugene Lavretsky, "Control of a Non affine DoublePendulum System via Dynamic Inversion and Time-Scale Separation", Proceedings of the 2006 American Control Conference Minneapolis, Minnesota, USA, June 2006.

[24] XinXin, "Analysis of the Energy Based Swing-up Control for a Double Pendulum on a Cart", In Proceedings of the 17th World Congress on the International Federation of Automatic Control Seoul, Korea, July 2008.

[25] Anita Khosla, Leena G and M. K. Soni, "ABC Algorithm Based Fuzzy Controller to Control the Velocity and Angle of an Inverted Pendulum", European Journal of Scientific Research, Vol.110, No.4, pp.493-500, 2013

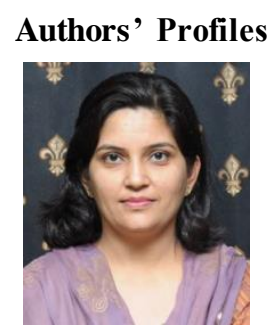

Ms. Anita Khosla has B-Tech in Electrical Engg. From NIT Kurukshetra and M-Tech in ECE in 1993and 2007 respectively. She is pursuing her $\mathrm{PhD}$ in control systems from ManavRachna International University, India. She had over 18 years of teaching experience and presently she is working as Associate Professer in the Electrical and Electronics Department of ManavRachna International University, Faridabad, India.

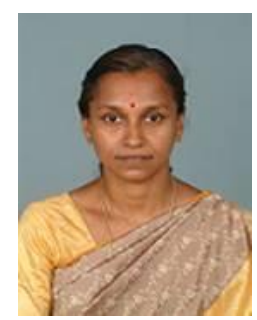

Dr. Leena G. has B-Tech in Electrical and Electronics Engg. and M-Tech in Control Systems from Kerala University in 1991and 1995 respectively. She completed her $\mathrm{PhD}$ in 2007 from Indian Institute of Technology, Kharagpur, India. She had over 15 years of teaching experience and presently she is professor in the Electrical and Electronics Department of ManavRachna International University, Faridabad, India. Her areas of interest are nonlinear system, decentralized control, sliding mode control etc.

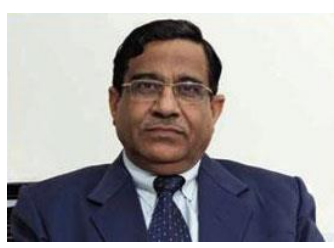

Dr.M.K.Soni has B.Sc.Engg in Electrical and MSC. Engg (Advanced Electronic \& Control System) fromR.E.C. Kurukshetra in 1972 and 1975 respectively. He completed his Phd. from R.E.C Kurukshetra in collaboration with IIT Delhi in 1988.He has experience of 39 years which includes teaching, research and administration. At present he is working as Executive Director \& Dean of ManavRachna International University, Faridabad, India. He has also worked as Director Principal of C.R.State college of EnggMurthal and as Professor \& Chairman in NIT Kurukshetra. His area of interests are Microprocessor, Microcontroller and Control Sy stems. Dr. Soni has guided 39 M.Tech Dissertation and and $7 \mathrm{PhDs}$. Presently 7 $\mathrm{PhDs}$ are under progress under his guidance. Dr. Soni is life time member of ISTE, System Society of India\&IASTED(Indian association of Science and Technology for Development).He is also Senior member of IEEE society.
How to cite this paper: Anita Khosla, Leena G., M. K. Soni,"Interval Type-2 Fuzzy Logic Controller to Control the Velocity and Angle of Inverted Pendulum", International Journal of Intelligent Systems and Applications(IJISA), vol.6, no.7, pp.44-51, 2014. DOI: 10.5815/ijisa.2014.07.06 